\title{
Deep Sclerectomy with Low-Tension Sutures versus Tight-Tension Sutures without Space-Maintaining Implant in Controlling Intraocular Pressures
}

\author{
Rashid Zia ${ }^{1}$, Nikhil Jain ${ }^{2}$, Albena Dardzhikova ${ }^{3}$ \\ 1,3 East Kent Hospitals University NHS Foundation Trust, Ashford, United Kingdom \\ ${ }^{2}$ University College London Hospitals NHS Foundation Trust, London, United Kingdom
}

\begin{abstract}
Purpose: To compare the IOP lowering effect and post-operative complications of low tension sutures versus tight tension sutures in deep sclerectomy.
\end{abstract}

Study Design: Quasi experimental study.

Place and Duration of Study: William Harvey Hospital, East Kent Hospitals University NHS Foundation Trust from 2015 to 2016 and Kent and Canterbury Hospital, East Kent Hospitals University NHS Foundation Trust from 2016 to 2017.

Methods: Twenty-seven eyes undergoing deep sclerectomy operation with normal strength sutures (TS) were compared with 21 eyes undergoing a modified technique in which low tension sutures were tied to only approximate anatomy (LS). Both sets of patients were operated on by the same experienced surgeon with similar technique except for the tensile strength of suture; same anti-metabolite in similar concentration was used with identical pre and post-operative care. Outcome measures were post-operative intraocular pressure, number of medications needed and complications.

Results: IOP decreased significantly in both groups, in TS group by $12.6 \mathrm{~mm} \mathrm{Hg}(p<0.001)$ and in the LS groups by $18.9 \mathrm{~mm} \mathrm{Hg}(p<0.001)$. Greater IOP drop was seen in LS group versus TS group $(p=0.046)$. In LS group number of eye drops required post operatively were significantly less than TS group $(p=0.007)$. After follow up only one patient in the loose suture group still required IOP-lowering medication. There were minimal complications in both groups, 1 patient in the LS group and 7 in TS group required a YAG Goniotomy.

Conclusion: Using low tension sutures in deep sclerectomy increases the IOP lowering effect of the surgery and reduces the number of medications the patient requires post operatively.

Key Words: Deep sclerectomy; Glaucoma; Sclerostomy, Trabeculectomy.

How to Cite this Article: Rashid Z, Jain N, Dardzhikova A. Comparison of Deep Sclerectomy with Low Tension Sutures Versus Tight - Tension Sutures Without Space - Maintaining Implant in Controlling Intraocular Pressures. Pak J Ophthalmol. 2020; 36 (4): 318-323.

Doi: https://doi.org/10.36351/pjo.v36i4.1031

Correspondence: Rashid Zia

Leads New Hayesbank Ophthalmology Services

Ashford Kent United Kingdom

Email: rashidzia@nhs.net

Received: March 23, 2020

Accepted: May 21, 2020

\section{INTRODUCTION}

Recent developments in glaucoma surgery have moved towards non-penetrating surgical techniques. Deep Sclerectomy (DS) has emerged as one of the more established of these procedures. There is ever increasing evidence to support its efficacy and safety profile with the latter being largely accepted as superior to many other alternatives ${ }^{1}$. This procedure 
entails removing the inner wall of Schlemm's canal and juxta-canalicular trabecular meshwork and leaving intact a trabeculo-Descemet's membrane (TDM) to control aqueous outflow through a filtration site $^{2}$. The initial steps of the procedure involve creating a superficial and deep scleral flap. The TDM is created by extending the dissection used to create the deep flap while also gently detaching Descemet's membrane. Once the dissection is complete, the deep flap is excised and the inner wall of Schlemm's canal and the juxta canalicular trabecular meshwork if peeled off. The superficial scleral flap is then attached using 10/0 nylon sutures ${ }^{2}$. In DS aqueous primarily drains through the anterior trabeculum and peripheral Descemet's membrane ${ }^{3,4}$. In addition, after aqueous humor has percolated through the TDM, it reaches a scleral lake formed at the site of the sclerectomy, under the superficial scleral flap. This artificial space may act as a first, intrascleral filtration bleb. From there, studies have shown some degree of aqueous drainage occurs into the sub-choroidal space and as well as through the subconjunctival pathway into the filtration bleb $^{5}$.

Traditionally, to keep the artificial intrascleral space patent, a space maintaining implant may be inserted in the scleral bed. Superficial scleral flap sutures tend to be tight to allow for small egress of fluid. We present data on a modified deep sclerectomy (MDS) whereby the operating steps are kept identical, but the sutures used to seal the superficial scleral flap are tied with less tension to approximate anatomy only.

\section{METHODS}

The patients were selected by convenient sampling from William Harvey Hospital, East Kent Hospitals University NHS Foundation Trust from 2015 to 2016 and Kent and Canterbury Hospital, East Kent Hospitals University NHS Foundation Trust from 2016 to 2017. Two groups of patients were analysed. Twenty-seven eyes, that were operated on at one site, formed the 'tight suture' (TS) group of patients; these patients had their procedure carried out according to the standard deep sclerectomy with normal strength sutures which allowed only a small egress of fluid. This data was contrasted against 21 eyes operated on at a second hospital, in which loose sutures were applied to approximate the anatomical structures; this formed the 'loose suture' (LS) group.
The sample sizes for both groups in our study was calculated as 19 participants for both groups by online RSS research calculator while taking in account the primary end point of the study.

All patients were operated under sub-tenon anaesthesia. Topical glaucoma therapy was stopped 1 week before surgery to optimise the ocular surface for surgery. None of the patients had cataract surgery before or during the observation period of the study. Intraocular pressures were controlled by systemic carbonic anhydrase inhibitors in the meantime. Approximation of structures was done using 10/0 nylon sutures (Ethilon, $\mathrm{J} \& \mathrm{~J}, \mathrm{USA}$ ) in all patients and no spacer devices were used in either group. Both sets of patients had 5Fluorourasil (5-FU) $50 \mathrm{mg} / \mathrm{ml}$, for 4 minutes on sponges before creating the superficial flap, as an anti-metabolite adjunct to the procedure. Post-operatively, all patients had 2 hourly preservative free $0.1 \%$ dexamethasone eye drops tailed off over 2 months along with preservative free chloramphenicol eye drops for 2 weeks and use of preservative free $0.15 \%$ Sodium Hyaluronate eye drops QDS.

The primary outcome measure was change in intra-ocular pressure (IOP). This was measured at 1day, 1 week, 1 month, 6-months and 1-year post operatively. However, for comparison, only 6 month and 1 year readings were analysed. The secondary outcome measure was the number of medications required by the patient post operatively to control their IOP. The tertiary outcome measures were the percentage of patients who had an IOP of less than $18 \mathrm{~mm} \mathrm{Hg}$, less than $21 \mathrm{~mm} \mathrm{Hg}$ at follow up and an IOP decrease of $30 \%$ or more.

The patients were also followed up to see if there were any complications after the procedure and these were recorded as per recommendations of "Guidelines on Design and Reporting Glaucoma Trials" published by World Glaucoma Association. In addition to this it was assessed how many patients required a YAG Goniotomy procedure post operatively.

Multiple statistical tests were used using SPSS version 23 to compare groups. A paired t- test was applied for comparison within each group. An unpaired t-test was done to find out any significant change in IOP between the groups. Chi-square test was used to assess significance for categorical data. A $p$ value of $<0.05$ was considered significant. 


\section{RESULTS}

Table 1 highlights the baseline IOP and medications of both groups. There was no significant difference in these characteristics. There were no significant differences in medications required, IOP measurements or complications at 1 year versus 6 months in either group. In both sets of patients the treatment regimen and disease had stabilised by 6 months. The data was analysed at 1 year follow-up.

Both treatments reduced the mean IOP in the respective groups; the TS group by $12.6 \mathrm{~mm} \mathrm{Hg}$ $(\mathrm{p}<0.001)$ and the LS group by $18.9 \mathrm{~mm} \mathrm{Hg}(\mathrm{p}<$ 0.001 ). In addition, both treatments reduced the number of drugs required to control IOP; by 1.8 (p < $0.001)$ in the TS group and by $3.6(\mathrm{p}<0.001)$ in the LS group. (As summarised in table 1).

When these means were contrasted between the groups, the difference in IOP drop and the reduction in drugs was significant $(\mathrm{p}<=0.046$ and $\mathrm{p}=0.007$ respectively). This is highlighted in table 2 .

Therefore, it can be inferred that each treatment reduces both the IOP and number of drugs required to control IOP after the operation and both of these effects are more substantial in the loose suture group.

Tertiary outcome measures were equivocal between the groups. In the TS group 26 out of 27 patients (96\%) had a follow up IOP of less than $18 \mathrm{~mm}$ $\mathrm{Hg}$ and as well as less than $21 \mathrm{~mm} \mathrm{Hg}$ versus all 21 $(100 \%)$ patients in the LS group (p-value 0.89). IOP reduction of greater than $30 \%$ occurred in $24 / 27$ (89\%) patients of TS group and in 19/21 (90\%) patients of the LS group (p-value 0.92). Therefore, there was no significant difference in the number of patients that had an IOP drop of more than $30 \%$ or a final IOP less than 18 or $21 \mathrm{~mm} \mathrm{Hg}$. As number of patients with IOP of less than $18 \mathrm{~mm} \mathrm{Hg}$ were identical to those with number of patients with $21 \mathrm{~mm} \mathrm{Hg}$ or less hence only one group was used for statistical analysis. However, it should be noted that in the LS group this pressure was maintained without medication in almost all patient whereas eye drops were still required to control IOP in the TS group.

In both groups the average number of drugs required pre-operatively was between 3 and 4 . Of the 27 eyes in the TS group, 8 eyes required no drugs for IOP post-operatively while 19 eyes still required some medication. Of the 21 eyes in the LS group 19 required no medication at the last follow up and only 2 patients still needed eye drops to maintain IOP within range. There were no cases in either group requiring more drugs than before the operation. However, statistical testing showed no significance in the number of patients requiring 'no drugs' versus 'fewer drugs' between groups $(\mathrm{p}=0.197)$. This means that we can confidently say that both procedures reduce the number of dugs required by patients and those with loose sutures require fewer drugs; we cannot confidently say that loosening sutures will leave patients requiring no drugs post operatively.

There were very few complications recorded in either group. In the LS group, one patient had corneal abrasion post operatively which healed with treatment and 1 needed a YAG goniotomy procedure. In the TS group, 2 patients were reported to have hyphaema and 7 patients needed a YAG goniotomy procedure 1

Table 1: Summary of the change in IOP and drugs required within each group at 1-year follow up. P-values show that both parameters decreased significantly in the groups.

\begin{tabular}{|c|c|c|c|c|c|c|}
\hline Technique & $\begin{array}{l}\text { Pre-operative } \\
\text { IOP }(\mathrm{mm} \mathrm{Hg})\end{array}$ & $\begin{array}{l}\text { Post-operative } \\
\text { IOP (mm Hg) }\end{array}$ & $P$ value & $\begin{array}{c}\text { Pre-operative } \\
\text { Medications }\end{array}$ & $\begin{array}{c}\text { Post-operative } \\
\text { Medications }\end{array}$ & P-value \\
\hline Tight Sutures & 28.3 & 15.5 & $<0.001$ & 3.1 & 1.26 & $<0.001$ \\
\hline Loose Sutures & 31.8 & 12.5 & $<0.001$ & 3.3 & 0.2 & $<0.001$ \\
\hline
\end{tabular}

Table 2: Mean difference in IOP and drugs required within each group and the significance of the difference between groups. Also shows what percentage of patients had IOP less than $18 \mathrm{mmHg}$ at follow up and a drop of $30 \%$ or more.

\begin{tabular}{|c|c|c|c|c|}
\hline Outcomes & Tight Sutures & Loose Sutures & Difference Between Groups & p value \\
\hline Decrease in IOP (mm Hg) & 12.6 & 19.3 & 6.3 & 0.046 \\
\hline Decrease in medications required & 1.8 & 3.1 & 1.3 & 0.007 \\
\hline $\begin{array}{l}\text { Percentage of patients with IOP below } 18 \mathrm{~mm} \\
\mathrm{Hg} \text { at follow-up }\end{array}$ & $96 \%$ & $100 \%$ & $4 \%$ & 0.89 \\
\hline $\begin{array}{l}\text { Percentage of patients with IOP drop of more } \\
\text { than } 30 \% \text { at follow-up }\end{array}$ & $89 \%$ & $90 \%$ & $1 \%$ & 0.92 \\
\hline
\end{tabular}

Pakistan Journal of Ophthalmology, 2020, Vol. 36 (3): 318-323 
month after the operation. None of the patients had postoperative hypotony, leaking bleb or iris incarceration. Cataract progression and endothelial cell loss were not observed. Requirement for a YAG goniotomy post operatively is not widely considered a complication of deep sclerectomy. It is more commonly thought of as a potential second step in a 2step operation; therefore, we did not include it as a complication. Hence, there appears to be no long term effects in any of our patients.

\section{DISCUSSION}

Adjunctive techniques are often used to increase the efficacy of DS. These include using intraoperative metabolites $^{6}$, collagen implants ${ }^{7}$ and YAG Goniotomy, should IOP rise above the target range. Given that, YAG goniopuncture can be considered as an adjunct to DS and we did not consider it a complication. ${ }^{8.9}$ It is nonetheless significant that only one case of the LS group required the procedure while just under a third of the TS group went on to the need of adjunctive procedure. According to some studies, the need for goniopuncture may be required in almost $40-60 \%$ of cases $^{8,9}$. While it has been shown that complication rates are low after $\mathrm{DS}^{4}$ our data would appear to suggest that these may be lower if loose sutures are applied, we likely need to look at a larger sample size to say this with confidence.

The widely accepted marker of success in glaucoma treatments is IOP less than $21 \mathrm{~mm} \mathrm{Hg}$ or a decrease in IOP of $30 \%$ or greater. Historically, the standard IOP control of $21 \mathrm{~mm} \mathrm{Hg}$ is considered as the upper limit of the normal IOP distribution from population-based studies and is used as cut off point ${ }^{10}$. In 1996, the 5 Fluorouracil Filtration surgery study group (5-FU study) $)^{11}$ used this value as the cut off point for the upper limit of normal. It further categorized success and failure based on the need for postoperative supplemental medication, reoperation and sight threatening complications. "Complete success" being defined as IOP of $21 \mathrm{~mm} \mathrm{Hg}$ or lower without medication, reoperation or devastating complications. Whereas "Qualified success" was reserved for IOP of $21 \mathrm{~mm} \mathrm{Hg}$ or lower with supplemental medication but without devastating complication. Recent clinical trials have adopted lower IOP cut off points of $<18$ or $<15 \mathrm{~mm} \mathrm{Hg}$ including Advanced Glaucoma intervention study (AGIS) ${ }^{12}$, the Collaborative Initial Glaucoma Treatment Study
$(\mathrm{CIGTS})^{13}$, the early manifest Glaucoma trial $(\mathrm{EMGT})^{14}$, and the Collaborative Normal Tension Glaucoma study ${ }^{15}$. The Association of international Glaucoma societies published consensus series in 2005 which report life-table results for annual IOP control under $18 \mathrm{~mm} \mathrm{Hg}$ for early damage and $15 \mathrm{~mm} \mathrm{Hg}$ for moderate to advance damage ${ }^{10}$. These varying criteria reflect the fact that different eyes require different degrees of IOP lowering based on baseline untreated IOP, the degree of glaucoma damage and other factors (trajectory of other eye, family history, life expectancy etc). Thus, any broadly applied IOP success criterion has limitations. Our data would appear to show that there are no differences in either of these measures at follow up and therefore in terms of clinical benefit to the patients these two techniques are comparable.

Success in this technique comes from the total reduction in IOP as well as the total cost of treating glaucoma and patient satisfaction and compliance. Post-operative IOP and post-operative drugs needed decreased significantly in the LS group versus the TS group. The reduction in drugs required to control IOP is perhaps most striking. Patients that undertake a regular deep sclerectomy operation still needed on average 2 different medications to be taken daily whereas in the LS group only 2 patients required eye drops at all. This has significant implications to patient satisfaction.

The long-term ramifications of needing to take drops versus not doing so should not be underestimated. Studies suggest that non-compliance or poor compliance with lifelong drops can range from $25-50 \%$ in patients with glaucoma ${ }^{16-18}$. Studies with longer followup show poor adherence with drops in patients that have the deep sclerectomy surgery with tight suture and worsening of their glaucoma. Evidence suggests that the fewer eye drops a patient is on, the more likely they are to be adherent to their regimen ${ }^{17}$.

In addition to this, the total cost of treatment is reduced. Patients with the tight suture requires more drugs and hence a greater cost. Costs could also be reduced in terms of follow up appointments. All but one of LS patients were medication free at 6 months whereas the TS patients still required the same number of drugs postoperatively at a year as at 6 months. With implementation of the looser sutures technique it is likely that patients will need either fewer clinic follow ups or total appointments thereby also comparatively 
reducing the cost between groups. Costs are also lowered if fewer patients require a YAG goniotomy.

Future developments and improvements further to this adjustment could be sutureless deep sclerectomy. In a pilot study of 24 eyes followed up for a period of 6 months with a modified deep sclerectomy technique to eliminate sutures altogether found that patients had a statistically significant reduction in IOP without extra complications ${ }^{19}$. While this technique mirrors our data. This study in combination with our study would suggest that tying sutures tightly and securely has a limiting effect on IOP drop and on maintaining a low IOP.

Studies have shown that deep sclerectomy is inferior to trabeculectomy but modifications in this technique can provide favourable results ${ }^{20}$.

Smalls sample size was the major limitation of our study. Further studies involving multiple centres are required.

\section{CONCLUSION}

Using looser sutures to seal the superficial scleral flap towards the end of the standard deep sclerectomy operation increases the IOP reduction, decreases the number of drugs patients require and has no increased rate of complications. This has significant ramifications for patient satisfaction and total healthcare costs. There is little or no benefit to applying tight sutures in the deep sclerectomy technique.

\section{List of Abbreviations:}

\begin{tabular}{|l|l|}
\hline TS & Tight Suture Group \\
\hline LS & Loose Suture Group \\
\hline IOP & Intra-ocular Pressure \\
\hline YAG & Yttrium Aluminium Garnet \\
\hline DS & Deep sclerectomy \\
\hline TDM & Trabeculo-Descemets Membrane \\
\hline MDS & Modified Deep Sclerectomy \\
\hline
\end{tabular}

\section{REFERENCES}

1. Sarodia U, Shaarawy T, Barton K. Nonpenetrating glaucoma surgery: a critical evaluation. Curr Opin Ophthalmol. 2007; 18 (2): 152-158.

2. Varga Z, Shaarawy T. Deep sclerectomy: safety and efficacy. Middle East Afr J Ophthalmol. 2009; 16 (3): 123-126.
3. Vaudaux J, Mermoud A. Aqueous humor dynamics in nonpenetrating filtering surgery. Ophthalmol Practice, 998; 38: 51064.

4. Mermoud A. Sinusotomy and deep sclerectomy. Eye (London). 2000; 14: 531-535.

5. Marchini G, Marraffa M, Brunelli C. Ultrasound biomicroscopy and intraocular pressure lowering mechanisms of deep sclerectomy with reticulated hyaluronic acid implant. J cataract Refract Surg. 2001; 27 (4): 507-517.

6. Kozobolis VP, Christodoulakis E V, Tzanakis N, Zacharopoulos I, Pallikaris IG. Primary deep sclerectomy versus primary deep sclerectomy with the use of mitomycin $\mathrm{C}$ in primary open-angle glaucoma. $\mathrm{J}$ Glaucoma. 2002; 11 (4): 287-293.

7. Karlen ME, Sanchez E, Schnyder CC, Sickenberg M, Mermoud A. Deep sclerectomy with collagen implant: medium term results. Br J Ophthalmol. 1999; 83 (1): 6-11.

8. Mendrinos E, Shaarawy T. The current situation in non-penetrating glaucoma surgery. Eur Ophthal Rev. 2009; 2 (1): 35-38.

9. Roy S, Mermoud A. Deep Sclerectomy. Dev Ophthalmol. 2012; 50: 29-36.

10. MO, Kass MA. The Ocular Hypertension Treatment study: Design and baseline description of the participants. Arch Ophthalmol. 1999; 117 (5): 573-583.

11. Garway-Heath DF, Quartilho A, Prah P, Crabb DP, Cheng Q, Zhu H. Evaluation of visual field and imaging outcomes for glaucoma clinical trials (an American Ophthalmological Society thesis). Trans Am Ophthalmol Soc. 2017; 115: T4.

12. The Advanced Glaucoma intervention study (AGIS): The relationship between control of intraocular pressure and visual filed deterioration. The AGIS investigators Am J Ophthalmology.2000;130(4):429-440.

13. Lichter PR, Musch DC, Gillespie BW, Guire KE, Janz NK, Wren PA, et al. Interim clinical outcomes in the Collaborative Initial Glaucoma Treatment Study comparing initial treatment randomized to medications or surgery. Ophthalmology. 2001;108(11):1943-1953.

14. Heijl A, Leske MC, Bengtsson B, Hyman L, Bengtsson B, Hussein M. Reduction of intraocular Pressure and Glaucoma progression: results from the Early Manifest Glaucoma Trial. Arch Ophthalmol. 2002:120(10):1268-1279.

15. The effectiveness of intraocular pressure reduction in the treatment of normal-tension glaucoma. Collaborative Normal-Tension Glaucoma Study Group. Am J Ophthalmol. 1998; 126 (4): 498-505.

16. Varga Z, Shaarawy T. Deep sclerectomy: safety and efficacy. Middle East Afr J Ophthalmol. 2009; 16 (3): 123-126. 
17. Nordmann J-P, Baudouin C, Renard J-P, Denis P, Regnault A, Berdeaux G. Identification of noncompliant glaucoma patients using Bayesian networks and the Eye-Drop Satisfaction Questionnaire. Clin Ophthalmol. 2010; 4: 1489-1496.

18. Konstas AGP, Maskaleris G, Gratsonidis S, Sardelli C. Compliance and viewpoint of glaucoma patients in Greece. Eye, 2000; 14 (5): 752-756. Doi: https://doi.org/10.1038/eye.2000.197

19. Abdelrahman AM, ElTanamly R, Sabry M. Sutureless Deep Sclerectomy: A Preliminary Report. J Glaucoma. 2017; 26 (11).

20. Sharifipour F, Yazdani S, Asadi M, Saki A, NouriMahdavi K. Modified Deep Sclerectomy for the Surgical Treatment of Glaucoma Yazdani. J Ophth Vis Res. 2019; 14: 144-150.

\section{Authors' Designation and Contribution}

Rashid Zia: Consultant Ophthalmologist; Study design, manuscript writing, final review.

Nikhil Jain: Specialty Trainee in Ophthalmology; Literature search, data analysis, manuscript preparation, manuscript editing, statistical analysis.

Albena Dardzhikova: Consultant Ophthalmologist, Literature search, manuscript preparation, manuscript editing, statistical analysis and review.

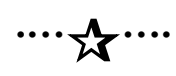

